\title{
Scholar Mirrors: Integrating evidence of impact from multiple sources into one platform to expedite researcher evaluation ${ }^{1}$
}

\author{
Alberto Martín-Martín*, Enrique Orduna-Malea** and Emilio Delgado López-Cózar* \\ *albertomartin@ugr.es; edelgado@ugr.es \\ EC3 Research Group, Universidad de Granada, Colegio Máximo de Cartuja, Granada, 18011 (Spain) \\ ** enorma@upv.es \\ EC3 Research Group, Universitat Politècnica de València, Camino de Vera s/n, Valencia, 46022 (Spain)
}

May 1, 2017

\begin{abstract}
This paper describes the creation of "Scholar Mirrors", a prototype web application that aims to provide a quick but accurate representation of the situation of a scientific discipline by integrating data from multiple online platforms. We chose the discipline of Bibliometrics / Scientometrics as a case study. After carrying out a series of keywords searches in Google Scholar Citations (GSC) and Google Scholar (GS), 813 relevant researchers were identified. Researchers were further classified as core (those who work mainly on Scientometrics) or related (those who work in other disciplines, with occasional incursions into Scientometrics). Additional information about these researchers was collected from other platforms (ResearcherID, ResearchGate, Mendeley, and Twitter). Up to 28 author-level indicators were collected about each researcher, as well as data about up to 100 of the most cited documents displayed in their GSC profile. The document-level data from all GSC profiles, as well as the data extracted from the keyword searchers in GS, was aggregated to create a list of the top 1000 most cited documents in the discipline. This document collection was further processed to generate a list of the most influential journals and publishers in the discipline. The results are accessible from the "Scholar Mirrors" website, which presents the results in four sections: authors, documents, journals, and book publishers. Lastly, the paper presents the main features of the web application, and the main limitations and future challenges of the product.
\end{abstract}

\section{KEYWORDS}

Google Scholar; ResearcherID; ResearchGate; Mendeley; Twitter; Author-level indicators; Web application

Important notice: this manuscript is a preprint and as such has not gone through a process of peer-review. Readers are encouraged to send their feedback via e-mail (address of corresponding author is available in the footer), Twitter (including the link to the article, or the authors' handles: @albertomartin, @eomalea, @GScholarDigest), or PubPeer.

\footnotetext{
${ }^{1}$ Alberto Martín-Martín enjoys a four-year doctoral fellowship granted by the Spanish Ministerio de Educación, Cultura, y Deporte (FPU2013/05863). Enrique Orduna-Malea holds a postdoctoral fellowship (PAID-10-14) from the Polytechnic University of Valencia (Spain).
} 


\section{INTRODUCTION}

In the last few years there has been a proliferation of platforms that enable researchers to disseminate their publications on the Web and track the degree to which they are used by other people. These for the most part previously unavailable indicators might become a good complement to the ones currently used in evaluative bibliometrics. These platforms, though similar in some ways, are very diverse as regards the sources of their data, their purpose for which they were designed, their features, their user base... all of which affects the impact indicators they present. Thus, each platform shows its own - more or less distorted - reflection of the performance of a researcher, not unlike what happens to a person who enters a house of mirrors attraction at an amusement park (hence the name "Scholar Mirrors").

The objective of this work is to present a prototype web application (Scholar Mirrors http://www.scholar-mirrors.infoec3.es) which collects mainly author-level indicators from several of these platforms for a specific community of researchers (in this case, the Bibliometrics/Scientometrics community). This study will address the issues of researcher selection, data collection and processing, and the design of the database and web interface used to visualize the data.

\section{METHODS}

The first task was to identify the set of researchers we wanted to study. To do this, we selected Google Scholar Citations (GSC) as our main source of data, and followed two different approaches so as to be as exhaustive as possible:

a) The keyword approach: A search was conducted in the most important journals of the field: Scientometrics, Journal of Informetrics, Research Evaluation, Cybermetrics, and the ISSI conferences (International Conference on Scientometrics and Informetrics) with the goal of extracting the most frequently used and representative words in the discipline. Table 1 shows the selected keywords. All public GSC profiles containing these keywords were selected. In addition, the lack of normalization in the use of keywords sometimes forced us to search variants of these keywords. These variants included misspelled words, the same keywords in other languages, etc. As an example, these are all the variants we found of the keyword "bibliometrics": bibliometric, bibliometría, bibliometria, bibliometric analysis, bibliometric methods, bibliometics, bibliometircs, bibliometric analysis in mining sciences, bibliometric mapping, bibliometric studies, bibliometric visualization, bibliometric., bibliometrics methodology, bibliometrics of social sciences and..., bibliometrics., bibliometrics..., bibliométrie, bibliometry.

b) The topic search approach: since there may be some authors working in this discipline who have created a public GSC profile, but who haven't added significant keywords or filled the institution field in their profile, we also conducted a topic search on Google Scholar (using the same keywords as before), and a journal search (all the documents indexed in Google Scholar published in the journals Scientometrics, Journal of Informetrics, Research Evaluation, Cybermetrics, as well as the ISSI conference proceedings), with the aim of finding authors we might have missed with the previous approach. This was possible because Google Scholar results display a link to the GSC profile of the author of the articles whenever a profile is available.

Table 1. Keywords selected to find authors

\begin{tabular}{|l|l|}
\hline Altmetrics & Research Assessment \\
\hline Bibliometrics & Research Evaluation \\
\hline
\end{tabular}




\begin{tabular}{|l|l|}
\hline Citation Analysis & Research Policy \\
\hline Citation Count & Science and Technology Policy \\
\hline H Index & Science Evaluation \\
\hline Impact Factor & Science Policy \\
\hline Informetrics & Science Studies \\
\hline Patent Citation & Scientometrics \\
\hline $\begin{array}{l}\text { Quantitative Studies of Science } \\
\text { and Technology }\end{array}$ & Webometrics \\
\hline
\end{tabular}

The searches were conducted on the $24^{\text {th }}$ of July, 2015. Researchers that didn't have a public GSC profile on that date are not included in this study.

Since Google Scholar Citations gives the author complete control over how to set their profile (personal information, institutional affiliation, research interests, as well as their scientific production), a systematic manual revision was carried out in order to:

a) Detect false positives: authors whose scientific production doesn't have anything to do with this discipline, even though they labelled themselves with one or more of the keywords associated with it.

b) Classify authors in two categories:

i. Core authors: those authors whose scientific production substantially falls within the field of Bibliometrics.

ii. Related authors: those authors who have sporadically published bibliometric studies, or whose field of expertise is closely related to Scientometrics (social, political, and economic studies about science), and therefore they can't be strictly considered bibliometricians.

In order to set a limit between the two categories, we decided to consider as core authors those who met the following criterion: at least half of the documents which contributed to their $h$ index had to be related to Bibliometrics. We considered the titles of the documents, as well as the publishing channel where they appeared, focusing our attention in the journals. Our Bradford-like core of journals about Bibliometrics consisted of six journals (Scientometrics, Journal of Informetrics, JASIST, Research Evaluation, Research Policy, Cybermetrics), followed by other LIS journals which also publish numerous bibliometric studies (Journal of Information Science, Information Processing \& Management, Journal of Documentation, College Research Libraries, Library Trends, Online Information Review, Revista Española de Documentación Científica, Aslib Proceedings, El Profesional de la Información) and lastly, journals devoted to social and political studies about science (Social Studies of Science, Science and Public Policy, Minerva, Journal of Health Services Research Policy, Technological Forecasting and Social Change, Science Technology Human Values, Environmental Science Policy, Current Science).

After this process, 813 relevant GSC profiles were identified. 397 of them were considered core authors, and the rest (416) as related authors. The data collection process was carried out using a custom web scraper written in Python. From each profile, this scraper extracted the researcher's personal information, all the author-level indicators available, and the bibliographic information of up to the 100 most cited documents in the profile, including the number of times cited. The data was initially saved as a two-table spreadsheet, one containing the personal information and author-level indicators for each author, and one containing the article references. 
These 813 authors were searched by name in ResearcherID, ResearchGate, Mendeley, and Twitter, and in the cases where a profile was found, the indicators provided by these platforms were downloaded. We selected these sources because they are the most popular and widely used (Van Noorden, 2015; Bosman \& Gramer, 2016). The data collection for these platforms was carried out between the 4th and 10th of September, 2015. Custom web scrapers were developed to extract the relevant author-level indicators from each platform.

A total of 28 author-level indicators were extracted from these sources:

a) Google Scholar Citations: sum of citations, h-index, and i10-index (for all years, and only for citations since 2010)

b) ResearchGate: RG Score, number of publications, sum of times cited, views, downloads, impact points, profile views, following (number of users the researcher follows), followers

c) Mendeley: number of publications, sum of readers, following, followers

d) ResearcherID (powered by Web of Science data): total number of articles in publication list, number of articles with citation data, sum of times cited, average citations per item, h-index

e) Twitter: number of tweets, days since registered, following, followers

Additionally, from the article data extracted from the GSC profiles, as well as from the articles found in the "topic search approach" to select authors, a list of the top 1000 most cited documents of the field according to Google Scholar was generated. The citation counts according to Web of Science (WoS) were also collected, thanks to the Google Scholar / Web of Science integration available to subscribing institutions. In the case of books and other materials which are not covered by WoS, manual searches were carried out using the cited reference search tool available in WoS.

Using this set of top of highly cited documents, rankings of the most relevant journals and book publishers in the discipline were generated (according to the percentage of articles/books published by each journal/book publisher in the sample).

Once all the data had been processed, the resulting tables were saved to a SQLite database using Python. This database is the core component of a custom web application written mostly in PHP (and a little bit of JavaScript for some components), available at http://www.scholarmirrors.infoec3.es. 


\section{DESCRIPTION OF THE WEB APPLICATION}

The application is structured in four sections: authors, documents, journals, and book publishers.

Figure 1. Screen capture of the authors section. General overview.

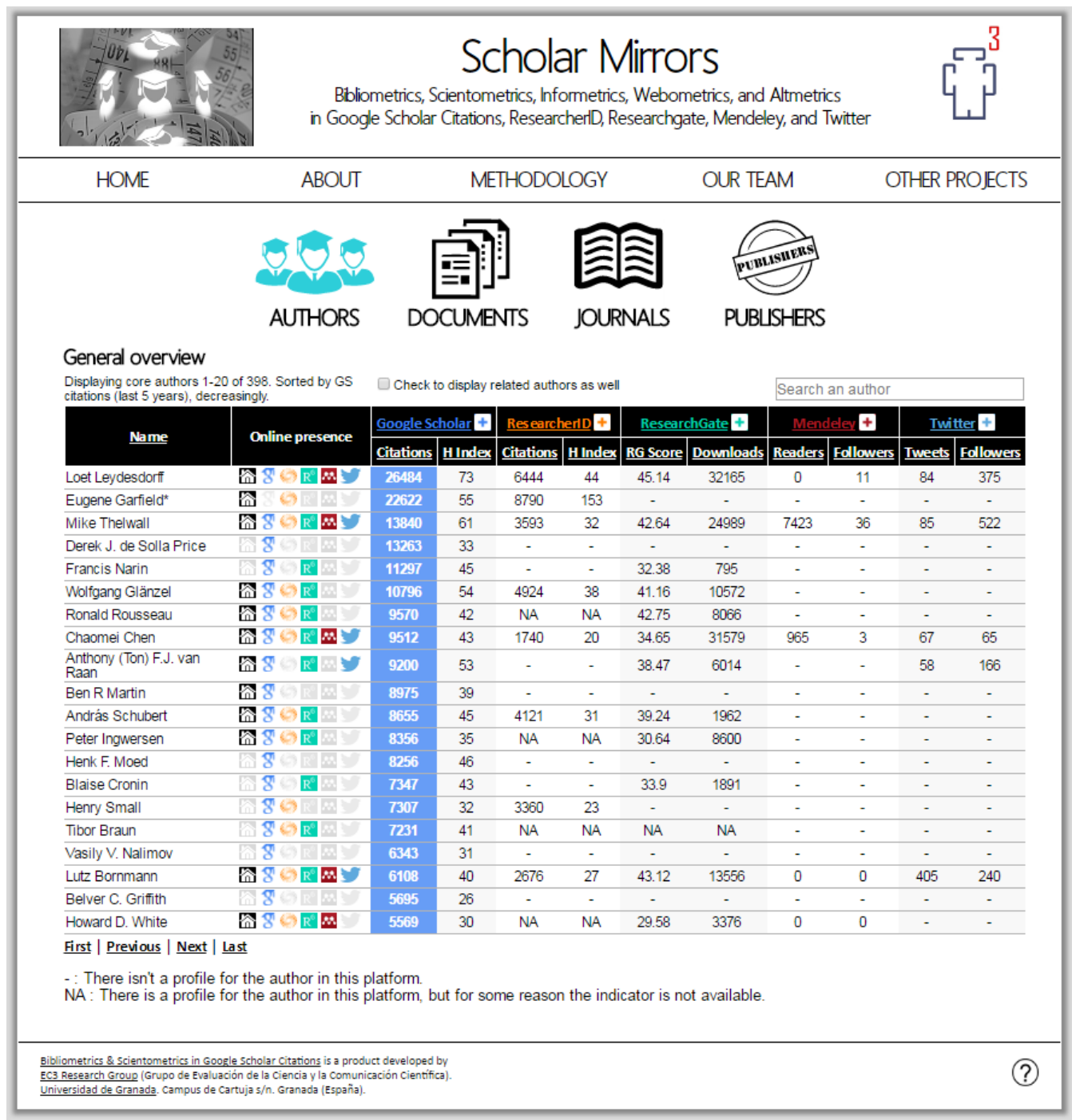

\section{Authors}

This is the main section of the application. By default, users are presented with a list of the top 20 most cited core authors in the field, according to Google Scholar (Figure 1). This is the General Overview page, and it displays the most relevant indicators available from each platform (Google Scholar, ResearcherID, ResearchGate, Mendeley, and Twitter), as well as a column called "Online presence" which presents the links to the profiles in those platforms, whenever available.

The navigation options in this page are the following:

a) Sorting tables: It is possible to sort the author tables by any of the displayed indicators, just by clicking on the name of the indicator. It is also possible to sort the table by names (alphabetically). By default, indicators will be sorted in descending order. When the table is sorted by a given indicator, clicking again in the name of the same indicator will sort the table in ascending order. Text fields will be sorted in ascending order by default. 
b) Navigating to following or previous pages: to facilitate visualization, only 20 authors are visible in each page. However, it is possible to navigate the entire set of authors by making use of the "First / Previous / Next / Last" links at the bottom-left side of the table.

c) Search Box: a search box is available to facilitate the task of finding a specific author. If a name or surname is entered in the box, a list of up to five names will appear just below it. Selecting one of them will automatically take the user to the page where that author is found (taking into account the current sorting criteria), and it'll be easily distinguishable from the rest because the background will be highlighted in yellow.

d) Navigating to platform-specific author tables: for each of the platforms there is a separate table that displays all the author indicators available in the platform. These tables can be accessed from the General Overview table by clicking in the appropriate header for each platform.

e) Core/Related authors: By default, only core authors are displayed. In order to display all authors, it is necessary to check the box with the label "Check to display related authors as well". When that box is checked, it'll be possible to tell core and related authors apart because the rows for core authors will be displayed with a grey background. Unchecking the box will hide related authors once again.

On the top-left part of the table a string of text will always inform of the current configuration parameters.

\section{Documents}

The documents section displays the top 1000 most cited documents in the field according to Google Scholar, along with the citation counts according to Web of Science. The bibliographic information in this table is structured in four columns: title of the document, authors, publication information (name of the journal, volume, issue, and pages in the case of journal articles, and publisher in the case of books or book chapters), and year of publication. This table can only be sorted by year of publication, times cited according to GS, and times cited according to WoS.

\section{Journals}

This section presents a ranking of journals according to the percentage of articles in the set of the top 1000 most cited documents in the field that are published in each journal. The percentage of citations of each journal (out of the sum of citations in those 1000 documents) is also displayed.

\section{Book Publishers}

This section presents a ranking of book publishers according to the percentage of books or book chapters in the set of the top 1000 most cited documents in the field that are published by each publisher. The percentage of citations of each book publisher (out of the sum of citations in those 1000 documents) is also displayed.

\section{LIMITATIONS AND FUTURE CHALLENGES}

As we advanced in the introduction, this product is only just an early prototype, with which we wanted to test the feasibility of developing a product that integrates impact indicators from diverse sources. We are aware of its many shortcomings, and believe that there is still a long way to go, if ever, before a product such as this one should be considered for use in evaluative processes.

The limitations we have detected are: 
- Only researchers with a public profile in Google Scholar Citations at the time of data collection are considered.

- The website is not easily updatable: the data collection scripts are not integrated into the web application. This means that a lot of human intervention is needed to update the data (running the web scrapers to extract the updated data from each platform, processing it, and adding the new data to the database). Ideally, these processes should be carried out automatically by the web application on a regular basis. When a more straightforward system is in place, it will also be possible to study the evolution of the indicators for a particular researcher over time.

- Incomplete or incorrect data in the profiles: most profile platforms leave on the hands of the users the responsibility of keeping their profile up to date and free of errors. However, many researchers don't consider this an important task, and so there are many outdated profiles, or profiles with incorrect information, which obviously affects the impact indicators. This issue is difficult to address, but at the very least, a few mechanisms to detect profiles that are likely to contain errors and warn about this should be implemented.

- Subject classification has been done manually. Ideally, an automatic classification, not at the author-level, but at the level of documents themselves, would allow a much more precise representation of the importance of an author in a specific area, field, or subfield. This is especially true in Bibliometrics, where researchers come from many different areas. A document-level classification would allow the calculation of author-level indicators using only the documents that are relevant to the field that is the object of study. The use of the core/related classification for authors in this product is just a rudimentary way of addressing this issue.

- The documents section (as well as the journals and book publishers sections) are just rough drafts of what could be done. In this product, the only sources of article-level indicators are Google Scholar and Web of Science. This could be extended to cover indicators from many other platforms (usage indicators, altmetrics...). Additionally, if more detailed information could be obtained, such as the references of the citing articles themselves, instead of only the citation counts, other issues like detecting unusual levels of self-citations could be addressed.

- Another aspect that this product doesn't address is collaboration. Only with the information available in Google Scholar it is possible to generate collaboration networks among researchers and in some cases even among institutions and countries, although richer metadata would probably be necessary to address the last two.

\section{REFERENCES}

Van Noorden, R. (2014). Online collaboration: Scientists and the social network. Nature, 512(7513), 126-129. https://doi.org/10.1038/512126a

Bosman J, Kramer B. (2016). Global survey on research tool usage. [Data set]. https://doi.org/10.5281/zenodo.49583 